Geometry $\&$ Topology

Volume 8 (2004) 311-334

Published: 14 February 2004

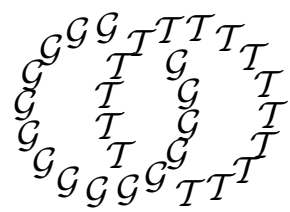

\title{
Holomorphic disks and genus bounds
}

\author{
Peter Ozsváth \\ Zoltán Szabó \\ Department of Mathematics, Columbia University \\ New York, NY 10025, USA and \\ Institute for Advanced Study, Princeton, New Jersey 08540, USA \\ and \\ Department of Mathematics, Princeton University \\ Princeton, New Jersey 08544, USA \\ Email: petero@math.columbia.edu and szabo@math.princeton.edu
}

\begin{abstract}
We prove that, like the Seiberg-Witten monopole homology, the Heegaard Floer homology for a three-manifold determines its Thurston norm. As a consequence, we show that knot Floer homology detects the genus of a knot. This leads to new proofs of certain results previously obtained using Seiberg-Witten monopole Floer homology (in collaboration with Kronheimer and Mrowka). It also leads to a purely Morse-theoretic interpretation of the genus of a knot. The method of proof shows that the canonical element of Heegaard Floer homology associated to a weakly symplectically fillable contact structure is non-trivial. In particular, for certain three-manifolds, Heegaard Floer homology gives obstructions to the existence of taut foliations.
\end{abstract}

AMS Classification numbers Primary: 57R58, 53D40

Secondary: $57 \mathrm{M} 27,57 \mathrm{~N} 10$

Keywords: Thurston norm, Dehn surgery, Seifert genus, Floer homology, contact structures

Proposed: Robion Kirby

Seconded: John Morgan, Ronald Stern
Received: 3 December 2003

Revised: 12 February 2004 


\section{Introduction}

The purpose of this paper is to verify that the Heegaard Floer homology of [27] determines the Thurston semi-norm of its underlying three-manifold. This further underlines the relationship between Heegaard Floer homology and SeibergWitten monopole Floer homology of [16], for which an analogous result has been established by Kronheimer and Mrowka, cf. [18.

Recall that Heegaard Floer homology $\underline{\widehat{H F}}(Y)$ is a finitely generated, $\mathbb{Z} / 2 \mathbb{Z}-$ graded $\mathbb{Z}\left[H^{1}(Y ; \mathbb{Z})\right]$-module associated to a closed, oriented three-manifold $Y$. This group in turn admits a natural splitting indexed by $\operatorname{Spin}^{c}$ structures $\mathfrak{s}$ over $Y$,

$$
\widehat{\widehat{H F}}(Y)=\bigoplus_{\mathfrak{s} \in \operatorname{Spin}^{c}(Y)} \underline{\hat{H F}}(Y, \mathfrak{s})
$$

(We adopt here notation from [27] the hat signifies here the simplest variant of Heegaard Floer homology, while the underline signifies that we are using the construction with "twisted coefficients", cf. Section 8 of [26].)

The Thurston semi-norm [39] on the two-dimensional homology of $Y$ is the function

$$
\Theta: H_{2}(Y ; \mathbb{Z}) \longrightarrow \mathbb{Z}^{\geq 0}
$$

defined as follows. The complexity of a compact, oriented two-manifold $\chi_{+}(\Sigma)$ is the sum over all the connected components $\Sigma_{i} \subset \Sigma$ with positive genus $g\left(\Sigma_{i}\right)$ of the quantity $2 g\left(\Sigma_{i}\right)-2$. The Thurston semi-norm of a homology class $\xi \in H_{2}(Y ; \mathbb{Z})$ is the minimum complexity of any embedded representative of $\xi$. (Thurston extends this function by linearity to a semi-norm $\Theta: H_{2}(Y ; \mathbb{Q}) \longrightarrow$ Q.)

Our result now is the following:

Theorem 1.1 The $\operatorname{Spin}^{c}$ structures $\mathfrak{s}$ over $Y$ for which the Heegaard Floer homology $\underline{\widehat{H F}}(Y, \mathfrak{s})$ is non-trivial determine the Thurston semi-norm on $Y$, in the sense that:

$$
\Theta(\xi)=\max _{\left\{\mathfrak{s} \in \operatorname{Spin}^{c}(Y) \mid \underline{\widehat{H F}}(Y, \mathfrak{s}) \neq 0\right\}}\left|\left\langle c_{1}(\mathfrak{s}), \xi\right\rangle\right|
$$

for any $\xi \in H_{2}(Y ; \mathbb{Z})$.

The above theorem has a consequence for the "knot Floer homology" of [31, [35. For simplicity, we state this for the case of knots in $S^{3}$. 
Recall that knot Floer homology is a bigraded Abelian group associated to an oriented knot $K \subset S^{3}$,

$$
\widehat{H F K}(K)=\bigoplus_{d \in \mathbb{Z}, s \in \mathbb{Z}} \widehat{H F K}_{d}(K, s)
$$

These groups are a refinement of the Alexander polynomial of $K$, in the sense that

$$
\sum_{s} \chi\left(\widehat{H F K}_{*}(K, s)\right) T^{s}=\Delta_{K}(T)
$$

where here $T$ is a formal variable, $\Delta_{K}(T)$ denotes the symmetrized Alexander polynomial of $K$, and

$$
\chi\left(\widehat{H F K}_{*}(K, s)\right)=\sum_{d \in \mathbb{Z}}(-1)^{d} \operatorname{rk} \widehat{H F K}_{d}(K, s),
$$

(cf. Equation 1 of [31]). One consequence of the proof of Theorem 1.1] is the following quantitative sense in which $\widehat{H F K}$ distinguishes the unknot:

Theorem 1.2 Let $K \subset S^{3}$ be a knot, then the Seifert genus of $K$ is the largest integer $s$ for which the group $\widehat{H F K}_{*}(K, s) \neq 0$.

This result in turn leads to an alternate proof of a theorem proved jointly by Kronheimer, Mrowka, and us [19], first conjectured by Gordon [13] (the cases where $p=0$ and \pm 1 follow from theorems of Gabai [9] and Gordon and Luecke [14] respectively):

Corollary 1.3 [19] Let $K \subset S^{3}$ be a knot with the property that for some integer $p, S_{p}^{3}(K)$ is diffeomorphic to $S_{p}^{3}(U)$ (where here $U$ is the unknot) under an orientation-preserving diffeomorphism, then $K$ is the unknot.

The first ingredient in the proof of Theorem 1.1 is a theorem of Gabai [8] which expresses the minimal genus problem in terms of taut foliations. This result, together with a theorem of Eliashberg and Thurston [5] gives a reformulation in terms of certain symplectically semi-fillable contact structures. The final breakthrough which makes this paper possible is an embedding theorem of Eliashberg [3], see also [6] and [25], which shows that a symplectic semi-filling of a three-manifold can be embedded in a closed, symplectic four-manifold. From this, we then appeal to a theorem [34, which implies the non-vanishing of the Heegaard Floer homology of a three-manifold which separates a closed, symplectic four-manifold. This result, in turn, rests on the topological quantum field-theoretic properties of Heegaard Floer homology, together with the 
suitable handle-decomposition of an arbitrary symplectic four-manifold induced from the Lefschetz pencils provided by Donaldson [2]. (The non-vanishing result from [34] is analogous to a non-vanishing theorem for the Seiberg-Witten invariants of symplectic manifolds proved by Taubes, cf. [36] and [37.)

\subsection{Contact structures}

In another direction, the strategy of proof for Theorem 1.1 shows that, just like its gauge-theoretic counterpart, the Seiberg-Witten monopole Floer homology, Heegaard Floer homology provides obstructions to the existence of weakly symplectically fillable contact structures on a given three-manifold, compare [17.

For simplicity, we restrict attention now to the case where $Y$ is a rational homology three-sphere, and hence $\widehat{H F}(Y) \cong \widehat{\widehat{H F}}(Y)$. In [30], we constructed an invariant $c(\xi) \in \widehat{H F}(Y)$, which we showed to be non-trivial for Stein fillable contact structures. In Section 4 we generalize this to the case of symplectically semi-fillable contact structures (see Theorem 4.2 for a precise statement). It is very interesting to see if this non-vanishing result can be generalized to the case of tight contact structures. (Of course, in the case where $b_{1}(Y)>0$, a reasonable formulation of this question requires the use of twisted coefficients, cf. Section 4 below.)

In Section 4 we also prove a non-vanishing theorem using the "reduced Heegaard Floer homology" $H F_{\text {red }}^{+}(Y)$ (for the image of $c(\xi)$ under a natural map $\widehat{H F}(Y) \longrightarrow H F_{\text {red }}^{+}(Y)$ ), in the case where $b_{2}^{+}(W)>0$ or $W$ is a weak symplectic semi-filling with more than one boundary component. According to a result of Eliashberg and Thurston [5], a taut foliation $\mathcal{F}$ on $Y$ induces such a structure.

One consequence of this is an obstruction to the existence of such a filling (or taut foliation) for a certain class of three-manifolds $Y$. An $L$-space 29] is a rational homology three-sphere with the property that $\widehat{H F}(Y)$ is a free $\mathbb{Z}$-module whose rank coincides with the number of elements in $H_{1}(Y ; \mathbb{Z})$. Examples include all lens spaces, and indeed all Seifert fibered spaces with positive scalar curvature. More interesting examples are constructed as follows: if $K \subset S^{3}$ is a knot for which $S_{p}^{3}(K)$ is an $L$-space for some $p>0$, then so is $S_{r}^{3}(K)$ for all rational $r>p$. A number of $L$-spaces are constructed in [29]. It is interesting to note the following theorem of Némethi: a three-manifold $Y$ is an $L$-space which is obtained as a plumbing of spheres if and only if it is the link of a rational surface singularity 24. L-spaces in the context of Seiberg-Witten monopole Floer homology are constructed in Section ( of 19] 
(though the constructions there apply equally well in the context of Heegaard Floer homology).

The following theorem should be compared with [20, 25] and [19] (see also [21]):

Theorem 1.4 An $L$-space $Y$ has no symplectic semi-filling with disconnected boundary; and all its symplectic fillings have $b_{2}^{+}(W)=0$. In particular, $Y$ admits no taut foliation.

\subsection{Morse theory and minimal genus}

Theorem 1.1 admits a reformulation which relates the minimal genus problem directly in terms of Morse theory on the underlying three-manifold. For simplicity, we state this in the case where $M$ is the complement of a knot $K \subset S^{3}$.

Fix a knot $K \subset S^{3}$. A perfect Morse function is said to be compatible with $K$, if $K$ is realized as a union of two of the flows which connect the index three and zero critical points (for some choice of generic Riemannian metric $\mu$ on $\left.S^{3}\right)$. Thus, the knot $K$ is specified by a Heegaard diagram for $S^{3}$, equipped with two distinguished points $w$ and $z$ where the knot $K$ meets the Heegaard surface. In this case, a simultaneous trajectory is a collection $\mathbf{x}$ of gradient flowlines for the Morse function which connect all the remaining (index two and one) critical points of $f$. From the point of view of Heegaard diagrams, a simultaneous trajectory is an intersection point in the $g$-fold symmetric product of $\Sigma$, $\operatorname{Sym}^{g}(\Sigma)$, (where $g$ is the genus of $\Sigma$ ) of two $g$-dimensional tori $\mathbb{T}_{\alpha}=$ $\alpha_{1} \times \ldots \times \alpha_{g}$ and $\mathbb{T}_{\beta}=\beta_{1} \times \ldots \times \beta_{g}$, where here $\left\{\alpha_{i}\right\}_{i=1}^{g}$ resp. $\left\{\beta_{i}\right\}_{i=1}^{g}$ denote the attaching circles of the two handlebodies.

Let $X=X(f, \mu)$ denote the set of simultaneous trajectories. Any two simultaneous trajectories differ by a one-cycle in the knot complement $M$ and hence, if we fix an identification $H_{1}(M ; \mathbb{Z}) \cong \mathbb{Z}$, we obtain a difference map

$$
\epsilon: X \times X \longrightarrow \mathbb{Z} \text {. }
$$

There is a unique map $s: X \longrightarrow \mathbb{Z}$ with the properties that $s(\mathbf{x})-s(\mathbf{y})=\epsilon(\mathbf{x}, \mathbf{y})$ for all $\mathbf{x}, \mathbf{y} \in X$, and also $\#\{\mathbf{x} \mid s(\mathbf{x})=i\} \equiv \#\{\mathbf{x} \mid s(\mathbf{x})=-i\}(\bmod 2)$ for all $i \in \mathbb{Z}$.

Although we will not need this here, it is worth pointing out that simultaneous trajectories can be viewed as a generalization of some very familiar objects from knot theory. To this end, note that a knot projection, together with a distinguished edge, induces in a natural way a compatible Heegaard diagram. The 
simultaneous trajectories for this Heegaard diagram can be identified with the "Kauffman states" for the knot projection; see [15] for an account of Kauffman states, and [33] for their relationship with simultaneous trajectories.

The following is a corollary of Theorem 1.1.

Corollary 1.5 The Seifert genus of a knot $K$ is the minimum over all compatible Heegaard diagrams for $K$ of the maximum of $s(\mathbf{x})$ over all the simultaneous trajectories.

It is very interesting to compare the above purely Morse-theoretic characterization of the Seifert genus with Kronheimer and Mrowka's purely differentialgeometric characterization of the Thurston semi-norm on homology in terms of scalar curvature, arising from the Seiberg-Witten equations, cf. [18. It would also be interesting to find a more elementary proof of the above result.

\subsection{Remark}

This paper completely avoids the machinery of gauge theory and the SeibergWitten equations. However, much of the general strategy adopted here is based on the proofs of analogous results in monopole Floer homology which were obtained by Kronheimer and Mrowka, cf. [18. It is also worth pointing out that although the construction of Heegaard Floer homology is completely different from the construction of Seiberg-Witten monopole Floer homology, the invariants are conjectured to be isomorphic. (This conjecture should be viewed in the light of the celebrated theorem of Taubes relating the Seiberg-Witten invariants of closed symplectic manifolds with their Gromov-Witten invariants, cf. 38.)

\subsection{Organization}

We include some preliminaries on contact geometry in Section 2 , and a quick review of Heegaard Floer homology in Section 3. In Section 4 , we prove the nonvanishing results for symplectically semi-fillable contact structures (including Theorem 1.4). In Section 5 we turn to the proofs of Theorems 1.1 and 1.2 and Corollaries 1.3 and 1.5. 


\subsection{Acknowledgements}

This paper would not have been possible without the fundamental new result of Yakov Eliashberg [3. We would like to thank Yasha for explaining his result to us, and for several illuminating discussions. We would also like to thank Peter Kronheimer, Paolo Lisca, Tomasz Mrowka, and András Stipsicz for many fruitful discussions. We would especially like to thank Kronheimer and Mrowka whose work in Seiberg-Witten monopole homology has served as an inspiration for this paper.

PSO was partially supported by NSF grant numbers DMS-0234311, DMS0111298, and FRG-0244663. ZSz was partially supported by NSF grant numbers DMS-0107792 and FRG-0244663, and a Packard Fellowship.

\section{Contact geometric preliminaries}

The three-manifolds we consider in this paper will always be oriented and connected (unless specified otherwise). A contact structure $\xi$ is a nowhere integrable two-plane distribution in $T Y$. The contact structures we consider in this paper will always be cooriented, and hence (since our three-manifolds are also oriented) the two-plane distributions $\xi$ are also oriented. Indeed, they can be described as the kernel of some smooth one-form $\alpha$ with the property that $\alpha \wedge d \alpha$ is a volume form for $Y$ (with respect to its given orientation). The form $d \alpha$ induces the orientation on $\xi$.

A contact structure $\xi$ over $Y$ naturally gives rise to a $\operatorname{Spin}^{c}$ structure, its canonical $\operatorname{Spin}^{c}$ structure, written $\mathfrak{k}(\xi)$, cf. [17. Indeed, Spin ${ }^{c}$ structures in dimension three can be viewed as equivalence classes of nowhere vanishing vector fields over $Y$, where two vector fields are considered equivalent if they are homotopic in the complement of a ball in $Y$, cf. [40], 12]. Dually, an oriented two-plane distribution gives rise to an equivalence class of nowhere vanishing vector fields (which are transverse to the distribution, and form a positive basis for $T Y$ ). Now, the canonical Spin ${ }^{c}$ structure of a contact structure is the $\operatorname{Spin}^{c}$ structure associated to its two-plane distribution. The first Chern class of the canonical $\operatorname{Spin}^{c}$ structure $\mathfrak{k}(\xi)$ is the first Chern class of $\xi$, thought of now as a complex line bundle over $Y$.

Four-manifolds considered in this paper are also oriented. A symplectic fourmanifold $(W, \omega)$ is a smooth four-manifold equipped with a smooth two-form $\omega$ satisfying $d \omega=0$ and also the non-degeneracy condition that $\omega \wedge \omega$ is a volume form for $W$ (compatible with its given orientation). 
Let $(W, \omega)$ be a compact, symplectic four-manifold $W$ with boundary $Y$. A four-manifold $W$ is said to have convex boundary if there is a contact structure $\xi$ over $Y$ with the property that the restriction of $\omega$ to the two-planes of $\xi$ is everywhere positive, cf. 4. Indeed, if we fix the contact structure $Y$ over $\xi$, we say that $W$ is a convex weak symplectic filling of $(Y, \xi)$. If $W$ is a convex weak symplectic filling of a possibly disconnected three-manifold $Y^{\prime}$ with contact structure $\xi^{\prime}$, and if $Y \subset Y^{\prime}$ is a connected subset with induced contact structure $\xi$, then we say that $W$ is a convex, weak semi-filling of $(Y, \xi)$. Of course, if a symplectic four-manifold $W$ has boundary $Y$, equipped with a contact structure $\xi$ for which the restriction of $\omega$ is everywhere negative, we say that $W$ has concave boundary, and that $W$ is a concave weak symplectic filling of $Y$. (We use the term "weak" here to be consistent with the accepted terminology from contact geometry. We will, however, never use the notion of strong symplectic fillings in this paper.)

If a contact structure $(Y, \xi)$ admits a weak convex symplectic filling, it is called weakly fillable. Note that every contact structure $(Y, \xi)$ can be realized as the concave boundary of some symplectic four-manifold (cf. [7], [10, and [3]). This is one justification for dropping the modifier "convex" from the terminology "weakly fillable". If a contact structure $(Y, \xi)$ admits a weak symplectic semifilling, then it is called weakly semi-fillable. According to a recent result of Eliashberg (cf. 3], restated in Theorem 4.1 below) any weakly semi-fillable contact structure is weakly fillable, as well.

A symplectic structure $(W, \omega)$ endows $W$ with a canonical $\operatorname{Spin}^{c}$ structure, denoted $\mathfrak{k}(\omega)$, cf. 36. This can be thought of as the canonical Spin ${ }^{c}$ struc- $^{-}$ ture associated to any almost-complex structure $J$ over $W$ compatible with $\omega$, compare [36. In particular, the first Chern class the $\operatorname{Spin}^{c}$ structure $\mathfrak{k}(\omega)$ is the first Chern class of its complexified tangent bundle. If $(W, \omega)$ has convex boundary $(Y, \xi)$, then the restriction of the canonical $\operatorname{Spin}^{c}$ structure over $W$ to $Y$ is the canonical $\operatorname{Spin}^{c}$ structure of the contact structure $\xi$.

\subsection{Foliations and contact structures}

Recall that a taut foliation is a foliation $\mathcal{F}$ which comes with a two-form $\omega$ which is positive on the leaves of $\mathcal{F}$ (note that like our contact structures, all the foliations we consider here are cooriented and hence oriented). An irreducible three-manifold is a three-manifold $Y$ with $\pi_{2}(Y)=0$. A fundamental result of Gabai states that if $Y$ is irreducible and $\Sigma_{0} \subset Y$ is an embedded surface which minimizes complexity in its homology class, and with has no spherical or 
toroidal components, then there is a smooth, taut foliation $\mathcal{F}$ which contains $\Sigma_{0}$ as a union of compact leaves. In particular, this shows that if $Y$ is an irreducible three-manifold with non-trivial Thurston semi-norm, and $\Sigma \subset Y$ is an embedded surface which minimizes complexity in its homology class, then there is a smooth, taut foliation $\mathcal{F}$ with the property that $\left\langle c_{1}(\mathcal{F}),[\Sigma]\right\rangle=-\chi_{+}(\Sigma)$. (Here, we let $\mathcal{F}$ be a taut foliation whose closed leaves include all the components of $\Sigma$ with genus greater than one.)

The link between taut foliations and semi-fillable contact structures is provided by an observation of Eliashberg and Thurston, cf. [5], according to which if $Y$ admits a smooth, taut foliation $\mathcal{F}$, then $W=[-1,1] \times Y$ can be given the structure of a convex symplectic manifold, where here the two-plane fields $\xi_{ \pm}$ over $\{ \pm 1\} \times Y$ are homotopic to the two-plane field of tangencies to $\mathcal{F}$.

\section{$3 \quad$ Heegaard Floer homology}

Heegaard Floer homology is a collection of $\mathbb{Z} / 2 \mathbb{Z}$-graded homology theories associated to three-manifolds, which are functorial under smooth four-dimensional cobordisms (cf. 27] for their constructions, and 28] for the verification of their functorial properties).

There are four variants, $\widehat{H F}(Y), H F^{-}(Y), H F^{\infty}(Y)$, and $H F^{+}(Y) \cdot H F^{-}(Y)$ is the homology of a complex over the polynomial ring $\mathbb{Z}[U], H F^{\infty}(Y)$ is the associated "localization" (i.e. it is the homology of the complex associated to tensoring with the ring of Laurent polynomials over $U), H F^{+}(Y)$ is associated to the cokernel of the localization map, and finally $\widehat{H F}(Y)$ is the homology of the complex associated to setting $U=0$. Indeed, all these groups admit splittings indexed by $\operatorname{Spin}^{c}$ structures over $Y$. The various groups are related by long exact sequences

$$
\begin{aligned}
& \ldots \longrightarrow \widehat{H F}(Y, \mathfrak{t}) \stackrel{i}{\longrightarrow} H F^{+}(Y, \mathfrak{t}) \stackrel{U}{\longrightarrow} H F^{+}(Y, \mathfrak{t}) \longrightarrow H F^{-} \longrightarrow \\
& \ldots \longrightarrow H F^{-}(Y, \mathfrak{t}) \stackrel{j}{\longrightarrow} H F^{\infty}(Y, \mathfrak{t}) \stackrel{\pi}{\longrightarrow} H F^{+}(Y, \mathfrak{t}) \longrightarrow \ldots,
\end{aligned}
$$

where here $\mathfrak{t} \in \operatorname{Spin}^{c}(Y)$. The "reduced Heegaard Floer homology" $H F_{\text {red }}^{+}(Y, \mathfrak{t})$ is the cokernel of the map $\pi$. Sometimes we distinguish this from $H F_{\text {red }}^{-}(Y, \mathfrak{t})$, which is the kernel of the map $j$, though these two $\mathbb{Z}[U]$ modules are identified in the long exact sequence above.

For $Y=S^{3}$, we have that $\widehat{H F}\left(S^{3}\right) \cong \mathbb{Z}$. We can now lift the $\mathbb{Z} / 2 \mathbb{Z}$ grading to an absolute $\mathbb{Z}$-grading on all the groups, using the following conventions. The 
group $\widehat{H F}\left(S^{3}\right) \cong \mathbb{Z}$ is supported in dimension zero, the maps $i, j$, and $\pi$ from Equation (11) preserve degree, and $U$ decreases degree by two. Indeed, for $S^{3}$, we have an identification of $\mathbb{Z}[U]$ modules:

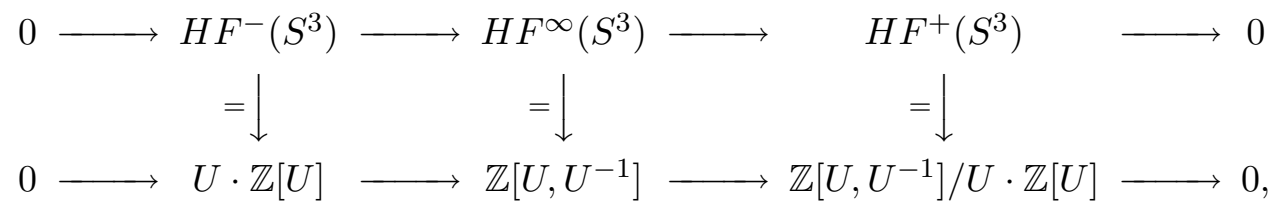

where here the element $1 \in \mathbb{Z}\left[U, U^{-1}\right]$ lies in grading zero and $U$ decreases grading by two. (See 32] for a definition of absolute gradings in more general settings.)

To state functoriality, we must first discuss maps associated to cobordisms. Let $W_{1}$ be a smooth, oriented four-manifold with $\partial W_{1}=-Y_{1} \cup Y_{2}$, where here $Y_{1}$ and $Y_{2}$ are connected. (Here, of course, $-Y_{1}$ denotes the three-manifold underlying $Y_{1}$, endowed with the opposite orientation.) In this case, we sometimes write $W_{1}: Y_{1} \longrightarrow Y_{2}$; or, turning this around, we can view the same fourmanifold as giving a cobordism $W_{1}:-Y_{2} \longrightarrow-Y_{1}$. There is an associated map

$$
\widehat{F}_{W_{1}}: \widehat{H F}\left(Y_{1}\right) \longrightarrow \widehat{H F}\left(Y_{2}\right),
$$

well-defined up to an overall multiplication by \pm 1 , which can be decomposed along $\operatorname{Spin}^{c}$ structures over $W_{1}$ :

$$
\widehat{F}_{W_{1}, \mathfrak{s}}: \widehat{H F}\left(Y_{1}, \mathfrak{t}_{1}\right) \longrightarrow \widehat{H F}\left(Y_{2}, \mathfrak{t}_{2}\right),
$$

where here $\mathfrak{t}_{i}=\left.\mathfrak{s}\right|_{Y_{i}}$, i.e. so that

$$
\widehat{F}_{W_{1}}=\sum_{\mathfrak{s} \in \operatorname{Spin}^{c}\left(W_{1}\right)} \widehat{F}_{W_{1}, \mathfrak{s}} .
$$

There are similarly induced maps $F_{W_{1, \mathfrak{s}}}^{+}$on $\mathrm{HF}^{+}$which are equivariant under the action of $\mathbb{Z}[U]$. For $H F^{\infty}$ and $H F^{-}$, there are again induced maps $F_{W_{1}, \mathfrak{s}}^{\infty}$ and $F_{W_{1}, \mathfrak{s}}^{-}$for each fixed $\operatorname{Spin}^{c}$ structure $\mathfrak{s} \in \operatorname{Spin}^{c}\left(W_{1}\right)$ (but now, we can no longer sum maps over all $\mathrm{Spin}^{c}$ structures, since infinitely many might be non-trivial). Indeed, these maps are compatible with the natural maps from Diagram (11); for example, all the squares in the following diagram commute:

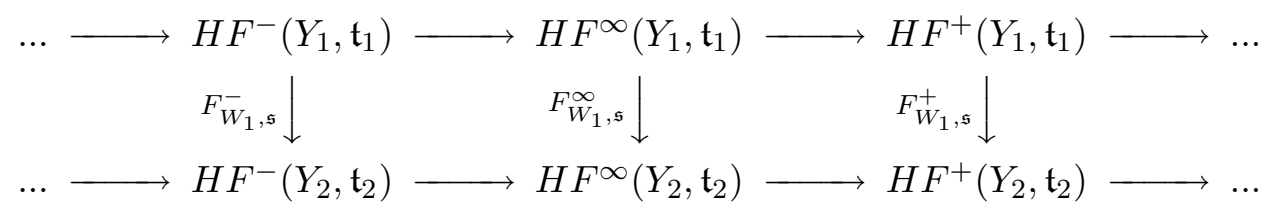


Functoriality of Floer homology is to be interpreted in the following sense. Let $W_{1}: Y_{1} \longrightarrow Y_{2}$ and $W_{2}: Y_{2} \longrightarrow Y_{3}$. We can form then the composite cobordism

$$
W_{1} \#_{Y_{2}} W_{2}: Y_{1} \longrightarrow Y_{3}
$$

We claim that for each $\mathfrak{s}_{i} \in \operatorname{Spin}^{c}\left(W_{i}\right)$ with $\left.\mathfrak{s}_{1}\right|_{Y_{2}}=\left.\mathfrak{s}_{2}\right|_{Y_{2}}$, we have that

$$
\sum_{\substack{\left.\left.\mathbb{F}_{Y_{2}} W_{2}\right) \\ \mathfrak{s}_{W_{i}}=\mathfrak{s}_{i}\right\}}} \widehat{F}_{W, \mathfrak{s}}=\widehat{F}_{W_{2}, \mathfrak{s}_{2}} \circ \widehat{F}_{W_{1}, \mathfrak{s}_{1}},
$$

with analogous formulas for $H F^{-}, H F^{\infty}$, and $H F^{+}$as well (this is the "composition law", Theorem 3.4 of [28] ).

Of these theories, $H F^{\infty}$ is the weakest at distinguishing manifolds. For example, if $W: Y_{1} \longrightarrow Y_{2}$ is a cobordism with $b_{2}^{+}(W)>0$, then for any $\operatorname{Spin}^{c}$ structure $\mathfrak{s} \in \operatorname{Spin}^{c}(W)$ the induced map

$$
F_{W, \mathfrak{s}}^{\infty}: H F^{\infty}\left(Y_{1},\left.\mathfrak{s}\right|_{Y_{1}}\right) \longrightarrow H F^{\infty}\left(Y_{2},\left.\mathfrak{s}\right|_{Y_{2}}\right)
$$

vanishes (cf. Lemma 8.2 of [28]).

Floer homology can be used to construct an invariant for smooth four-manifolds $X$ with $b_{2}^{+}(X)>1$ (here, $b_{2}^{+}(X)$ denotes the dimension of the maximal subspace of $H^{2}(X ; \mathbb{R})$ on which the cup-product pairing is positive-definite) endowed with a $\operatorname{Spin}^{c}$ structure $\mathfrak{s} \in \operatorname{Spin}^{c}(X)$

$$
\Phi_{X, \mathfrak{s}}: \mathbb{Z}[U] \longrightarrow \mathbb{Z},
$$

which is well-defined up to an overall sign. This invariant is analogous to the Seiberg-Witten invariant, cf. 41]. This map is a homogeneous element in $\operatorname{Hom}(\mathbb{Z}[U], \mathbb{Z})$ with degree given by

$$
\frac{c_{1}(\mathfrak{s})^{2}-2 \chi(X)-3 \sigma(X)}{4} .
$$

For a fixed four-manifold $X$, the invariant $\Phi_{X, \mathfrak{s}}$ is non-trivial for only finitely many $\mathfrak{s} \in \operatorname{Spin}^{c}(X)$. (Note that the four-manifold invariant $\Phi_{X, \mathfrak{s}}$ constructed in [28] is slightly more general, as it incorporates the action of $H_{1}(X ; \mathbb{Z})$, but we do not need this extra structure for our present applications.)

The invariant is constructed as follows. Let $X$ be a four-manifold, and fix a separating hypersurface $N \subset X$ with $0=\delta H^{1}(N ; \mathbb{Z}) \subset H^{2}(X ; \mathbb{Z})$, so that $X=X_{1} \cup_{N} X_{2}$, with $b_{2}^{+}\left(X_{i}\right)>0$ for $i=1,2$. (Here, $\delta: H^{1}(Y ; \mathbb{Z}) \longrightarrow$ $H^{2}(X ; \mathbb{Z})$ is the connecting homomorphism in the Mayer-Vietoris sequence for the decomposition of $X$ into $X_{1}$ and $X_{2}$.) Such a separating three-manifold 
is called an admissible cut in the terminology of [28]. Given such a cut, delete balls $B_{1}$ and $B_{2}$ from $X_{1}$ and $X_{2}$ respectively, and consider the diagram:

$$
\begin{aligned}
& H F^{-}\left(S^{3}\right) \longrightarrow H F^{\infty}\left(S^{3}\right) \\
& F_{X_{1}-B_{1}, \mathfrak{s}_{1}}^{-} \downarrow \quad F_{X_{1}-B_{1}, \mathfrak{s}_{1}}^{\infty} \downarrow 0 \\
& H F^{\infty}(N, \mathfrak{t}) \longrightarrow H F^{+}(N, \mathfrak{t}) \longrightarrow H F^{-}(N, \mathfrak{t}) \longrightarrow H F^{\infty}(N, \mathfrak{t}) \\
& 0 \downarrow F_{X_{2}-B_{2}, \mathfrak{s}_{2}}^{\infty} \quad \downarrow F_{X_{2}-B_{2}, \mathfrak{s}_{2}}^{+} \\
& H F^{\infty}\left(S^{3}\right) \longrightarrow H F^{+}\left(S^{3}\right),
\end{aligned}
$$

where here $\mathfrak{t}=\left.\mathfrak{s}\right|_{N}$ and $\mathfrak{s}_{i}=\left.\mathfrak{s}\right|_{X_{i}}$. Since the two maps indicated with 0 vanish (as $b_{2}^{+}\left(X_{i}-B_{i}\right)>0$ ), there is a well-defined map

$$
F_{X-B_{1}-B_{2}, \mathfrak{s}}^{\operatorname{mix}}: H F^{-}\left(S^{3}\right) \longrightarrow H F^{+}\left(S^{3}\right),
$$

which factors through $H F_{\text {red }}^{+}(N, \mathfrak{t})$.

The invariant $\Phi_{X, \mathfrak{s}}$ corresponds to $F_{X-B_{1}-B_{2}, \mathfrak{s}}^{\operatorname{mix}}$ under the natural identification

$$
\operatorname{Hom}_{\mathbb{Z}[U]}\left(\mathbb{Z}[U], \mathbb{Z}\left[U, U^{-1}\right] / \mathbb{Z}[U]\right) \cong \operatorname{Hom}(\mathbb{Z}[U], \mathbb{Z})
$$

According to Theorem 9.1 of $[28], \Phi_{X, \mathfrak{s}}$ is a smooth four-manifold invariant.

The following property of the invariant is immediate from its definition: if $X=$ $X_{1} \cup_{N} X_{2}$ where $N$ is a rational homology three-sphere with $H F_{\text {red }}^{+}(N)=0$, and the four-manifolds $X_{i}$ have the property that $b_{2}^{+}\left(X_{i}\right)>0$, then for each $\mathfrak{s} \in \operatorname{Spin}^{c}(X)$,

$$
\Phi_{X, \mathfrak{s}} \equiv 0 .
$$

The second property which we rely on heavily in this paper is the following analogue of a theorem of Taubes [36] and 37 for the Seiberg-Witten invariants for four-manifolds: if $(X, \omega)$ is a smooth, closed, symplectic four-manifold with $b_{2}^{+}(X)>1$, then if $\mathfrak{k}(\omega) \in \operatorname{Spin}^{c}(X)$ denotes its canonical $\operatorname{Spin}^{c}$ structure, then we have that

$$
\Phi_{X, \mathfrak{k}(\omega)} \equiv \pm 1
$$

while if $\mathfrak{s} \in \operatorname{Spin}^{c}(X)$ is any $\operatorname{Spin}^{c}$ structure for which $\Phi_{X, \mathfrak{s}} \not \equiv 0$, then we have that

$$
\left\langle c_{1}(\mathfrak{k}(\omega)) \cup \omega,[X]\right\rangle \leq\left\langle c_{1}(\mathfrak{s}) \cup \omega,[X]\right\rangle,
$$

with equality iff $\mathfrak{s}=\mathfrak{k}(\omega)$. This result is Theorem 1.1 of [34, and its proof relies on a combination of techniques from Heegaard Floer homology (specifically, the surgery long exact sequence from [26]) and Donaldson's Lefschetz pencils for symplectic manifolds, 2]. 


\subsection{Three-manifolds with $b_{1}(Y)>0$}

There is a version of Floer homology with "twisted coefficients" which is relevant in the case where $b_{1}(Y)>0$. Fundamental to this construction is a chain complex $\underline{\widehat{C F}}(Y)$ (and also corresponding complexes $\underline{C F^{-}}, \underline{C F^{\infty}}$, and $\underline{C F^{+}}$) with coefficients in $\mathbb{Z}\left[H^{1}(Y ; \mathbb{Z})\right]$ which is a lift of the complex $\widehat{C F}(Y)$ (whose homology calculates $\widehat{H F}(Y))$, in the following sense. Let $\mathbb{Z}$ be the module over $\mathbb{Z}\left[H^{1}(Y ; \mathbb{Z})\right]$, where the elements of $H^{1}(Y ; \mathbb{Z})$ act trivially. Then, there is an identification $\widehat{C F}(Y) \cong \widehat{\widehat{C F}}(Y) \otimes_{\mathbb{Z}\left[H^{1}(Y ; \mathbb{Z})\right]} \mathbb{Z}$. Thus, there is a change of coefficient spectral sequences which relates the homology of $\underline{\widehat{C F}}(Y)$, written $\widehat{H F}(Y)$, with $\widehat{H F}(Y)$.

Indeed, given any module $M$ over $\mathbb{Z}\left[H^{1}(Y ; \mathbb{Z})\right]$, we can form the group

$$
\underline{\widehat{H F}}(Y ; M)=H_{*}\left(\underline{\widehat{C F}}(Y) \otimes_{\mathbb{Z}\left[H^{1}(Y ; \mathbb{Z})\right]} M\right),
$$

which gives Floer homology with coefficients twisted by $M$. The analogous construction in the other versions of Floer homology gives groups $\mathrm{HF}^{-}(Y ; M)$, $\underline{H F}^{\infty}(Y ; M)$, and $\underline{H F}^{+}(Y ; M)$. All of these are related by exact sequences analogous to those in Diagram (11). In particular, we can form a reduced group $\underline{H F}+{ }_{\mathrm{red}}^{+}(Y ; M)$, which is the cokernel of the localization map $\underline{H F}^{\infty}(Y ; M) \longrightarrow$ $\underline{H F}^{+}(Y ; M)$.

In particular, if we fix a two-dimensional cohomology class $[\omega] \in H^{2}(Y ; \mathbb{R})$, we can view $\mathbb{Z}[\mathbb{R}]$ as a module over $\mathbb{Z}\left[H^{1}(Y ; \mathbb{Z})\right]$ via the ring homomorphism

$$
[\gamma] \mapsto T^{\int_{Y}[\gamma] \wedge \omega}
$$

(where here $T^{r}$ denotes the group-ring element associated to the real number $r)$. This gives us a notion of twisted coefficients which we denote by $\underline{\widehat{H F}}(Y ;[\omega])$. This can be thought of explicitly as follows. Choose a Morse function on $Y$ compatible with a Heegaard decomposition $(\Sigma, \boldsymbol{\alpha}, \boldsymbol{\beta}, z)$, and fix also a twococycle $\omega$ over $Y$ which represents $[\omega]$. We obtain a map from Whitney disks $u$ in $\operatorname{Sym}^{g}(\Sigma)$ (for $\mathbb{T}_{\alpha}$ and $\mathbb{T}_{\beta}$ ) to two-chains in $Y: u$ induces a two-chain in $\Sigma$ with boundaries along the $\boldsymbol{\alpha}$ and $\boldsymbol{\beta}$. These boundaries are then coned off by following gradient trajectories for the $\alpha$-and $\beta$-circles. Since $\omega$ is a cocycle, the evaluation of $\omega$ on $u$ depends only on the homotopy class $\phi$ of $u$. We denote this evaluation by $\int_{[\phi]} \omega$. (This determines an additive assignment in the terminology of Section 8 of [26].) The differential on $\underline{H F}^{+}(Y ;[\omega])$ is given by

$$
\underline{\partial}^{+}[\mathbf{x}, i]=\sum_{\mathbf{y} \in \mathbb{T}_{\alpha} \cap \mathbb{T}_{\beta}} \sum_{\left\{\phi \in \pi_{2}(\mathbf{x}, \mathbf{y}) \mid \mu(\phi)=1\right\}} \#\left(\frac{\mathcal{M}(\phi)}{\mathbb{R}}\right) \cdot T^{\int_{[\phi]} \omega} \cdot\left[\mathbf{y}, i-n_{z}(\phi)\right],
$$


where here we adopt notation from [26: $\pi_{2}(\mathbf{x}, \mathbf{y})$ denotes the space of homotopy classes of Whitney disks in $\operatorname{Sym}^{g}(\Sigma)$ for $\mathbb{T}_{\alpha}$ and $\mathbb{T}_{\beta}$ connecting $\mathbf{x}$ and $\mathbf{y}, \mu(\phi)$ denotes the formal dimension of its space $\mathcal{M}(\phi)$ of holomorphic representatives, and $n_{z}(\phi)$ denotes the intersection number of $\phi$ with the subvariety $\{z\} \times$ $\operatorname{Sym}^{g-1}(\Sigma) \subset \operatorname{Sym}^{g}(\Sigma)$.

Now, if $W: Y_{1} \longrightarrow Y_{2}$, and $M_{1}$ is a module over $H^{1}\left(Y_{1} ; \mathbb{Z}\right)$, there is an induced map

$$
\underline{F}_{W ; M_{1}}^{+}: \underline{H F}^{+}\left(Y_{1}, M_{1}\right) \longrightarrow \underline{H F}^{+}\left(Y_{2}, M_{1} \otimes_{H^{1}\left(Y_{1} ; \mathbb{Z}\right)} H^{2}\left(W, Y_{1} \cup Y_{2}\right)\right),
$$

well-defined up to the action by some unit in $\mathbb{Z}\left[H^{2}\left(Y_{1} \cup Y_{2} ; \mathbb{Z}\right)\right]$, defined as in Subsection 3.1 [28]. (Indeed, in that discussion, the construction is separated according to $\operatorname{Spin}^{c}$ structures over $W$, which we drop at the moment for notational simplicity.) In the case of $\omega$-twisted coefficients, this gives rise to a map

$$
\underline{F}_{W ;[\omega]}^{+}: \underline{H F}^{+}\left(Y_{1} ;\left.[\omega]\right|_{Y_{1}}\right) \longrightarrow \underline{H F}^{+}\left(Y_{2} ;\left.[\omega]\right|_{Y_{2}}\right)
$$

(again, well-defined up to multiplication by $\pm T^{c}$ for some $c \in \mathbb{R}$ ) which can be concretely described as follows.

Suppose for simplicity that $W$ is represented as a two-handle addition, so that there is a corresponding "Heegaard triple" $(\Sigma, \boldsymbol{\alpha}, \boldsymbol{\beta}, \boldsymbol{\gamma}, z)$. The corresponding four-manifold $X_{\alpha, \beta, \gamma}$ represents $W$ minus a one-complex. Fix now a two-cocycle $\omega$ representing $[\omega] \in H^{2}(W ; \mathbb{R})$. Again, a Whitney triangle $u$ in $\operatorname{Sym}^{g}(\Sigma)$ for $\mathbb{T}_{\alpha}, \mathbb{T}_{\beta}$, and $\mathbb{T}_{\gamma}$ (with vertices at $\mathbf{x}, \mathbf{y}$, and $\mathbf{w}$ ) determines a two-chain in $X_{\alpha, \beta, \gamma}$, whose evaluation on $\omega$ depends on $u$ only through its induced homotopy class $\psi$ in $\pi_{2}(\mathbf{x}, \mathbf{y}, \mathbf{w})$, denoted by $\int_{[\psi]} \omega$. Now,

$$
\underline{F}_{W ;[\omega]}^{+}[\mathbf{x}, i]=\sum_{\mathbf{y} \in \mathbb{T}_{\alpha} \cap \mathbb{T}_{\gamma}} \sum_{\left\{\psi \in \pi_{2}(\mathbf{x}, \Theta, \mathbf{y}) \mid \mu(\psi)=0\right\}} \#(\mathcal{M}(\psi)) \cdot T^{\int_{[\psi]} \omega} \cdot\left[\mathbf{y}, i-n_{z}(\psi)\right],
$$

where $\Theta \in \mathbb{T}_{\beta} \cap \mathbb{T}_{\gamma}$ represents a canonical generator for the Floer homology $H F \leq=H_{*}\left(U^{-1} \cdot \mathrm{C} F^{-}\right)$of the three-manifold determined by $(\Sigma, \boldsymbol{\beta}, \boldsymbol{\gamma}, z)$, which is a connected sum $\#^{g-1}\left(S^{2} \times S^{1}\right)$. This can be extended to arbitrary (smooth, connected) cobordisms from $Y_{1}$ to $Y_{2}$ as in [28].

(In the present discussion, since we have suppressed Spin ${ }^{c}$ structures from the notation, a subtlety arises. The expression analogous to Equation (3), only using $H F^{-}$, is not well-defined since, in principle, there might be infinitely many different homotopy classes which induce non-trivial maps - i.e. we are trying to sum the maps on $H F^{-}$induced by infinitely many different $\operatorname{Spin}^{c}$ structures. However, if the cobordism $W$ has $b_{2}^{+}(W)>0$, then there are 
only finitely many $\operatorname{Spin}^{c}$ structures which induce non-zero maps, according to Theorem 3.3 of $[28]$.)

Note that when $W$ is a cobordism between two integral homology three-spheres, the above construction is related to the construction in the untwisted case by the formula

$$
\underline{F}_{W ;[\omega]}^{+}= \pm T^{c} \cdot \sum_{\mathfrak{s} \in \operatorname{Spin}^{c}(W)} T^{\left\langle c_{1}(\mathfrak{s}) \cup[\omega],[W]\right\rangle} \cdot F_{W, \mathfrak{s}}^{+}
$$

for some constant $c \in \mathbb{R}$.

\section{Invariants of weakly fillable contact structures}

We briefly review the construction here of the Heegaard Floer homology element associated to a contact structure $\xi$ over the three-manifold $Y, c(\xi) \in \widehat{H F}(-Y)$. After sketching the construction, we describe a refinement which lives in Floer homology with twisted coefficients.

The contact invariant is constructed with the help of some work of Giroux. Specifically, in [1], Giroux shows that contact structures over $Y$ are in oneto-one correspondence with equivalence classes of open book decompositions of $Y$, under an equivalence relation given by a suitable notion of stabilization. Indeed, after stabilizing, one can realize the open book with connected binding, and with genus $g>1$ (both are convenient technical devices). In particular, performing surgery on the binding, we obtain a cobordism (obtained by a single two-handle addition) $W_{0}: Y \longrightarrow Y_{0}$, where here the three-manifold $Y_{0}$ fibers over the circle. We call this cobordism a Giroux two-handle subordinate to the contact structure over $Y$. This cobordism is used to construct $c(\xi)$, but to describe how, we must discuss the Heegaard Floer homology for three-manifolds which fiber over the circle.

Let $Z$ be a (closed, oriented) three-manifold endowed with the structure of a fiber bundle $\pi: Z \longrightarrow S^{1}$. This structure endows $Z$ with a canonical $\operatorname{Spin}^{c}$ structure $\mathfrak{k}(\pi) \in \operatorname{Spin}^{c}(Z)$ (induced by the two-plane distribution of tangents to the fiber of $\pi$ ). According to [34, if the genus $g$ of the fiber is greater than one, then

$$
H F^{+}(Z, \mathfrak{k}(\pi)) \cong \mathbb{Z}
$$

In particular, there is a homogeneous generator $c_{0}(\pi)$ for $\widehat{H F}(Z, \mathfrak{k}(\pi)) \cong \mathbb{Z} \oplus \mathbb{Z}$ which maps to the generator $c_{0}^{+}(\pi)$ of $H F^{+}(Z, \mathfrak{k}(\pi))$. This generator is, of course, uniquely determined up to sign. 
With these remarks in place, we can give the definition of the invariant $c(\xi)$ associated to a contact structure over $Y$. If $Y$ is given a contact structure, fix a compatible open book decomposition (with connected binding, and fiber genus $g>1$ ), and consider the corresponding Giroux two-handle $W_{0}:-Y_{0} \longrightarrow-Y$ (which we have "turned around" here), and let

$$
\widehat{F}_{W_{0}}: \widehat{H F}\left(-Y_{0}\right) \longrightarrow \widehat{H F}(-Y)
$$

be the induced map. Then, define $c(\xi) \in \widehat{H F}(-Y) /\{ \pm 1\}$ to be the image $\widehat{F}_{W_{0}}\left(c_{0}(\pi)\right)$. It is shown in [30] that this element is uniquely associated (up to sign) to the contact structure, i.e. it is independent of the choice of compatible open book. In fact, the element $c(\xi)$ is supported in the summand $\widehat{H F}(Y, \mathfrak{k}(\xi)) \subset \widehat{H F}(Y)$, where here $\mathfrak{k}(\xi)$ is the canonical Spin $^{c}$ structure associated to the contact structure $\xi$, in the sense described in Section 2 . (In particular, the canonical $\operatorname{Spin}^{c}$ structure of the fibration structure on $-Y_{0}$ is $\mathrm{Spin}^{c}$ cobordant to the canonical $\mathrm{Spin}^{c}$ structure of the contact structure over $-Y$ via the Giroux two-handle.)

With the help of Giroux's characterization of Stein fillable contact structures, it is shown in 30] that $c(\xi)$ is non-trivial for a Stein structure. This non-vanishing result can be strengthened considerably with the help of the following result of Eliashberg [3].

Theorem 4.1 (Eliashberg [3]) Let $(Y, \xi)$ be a contact three-manifold, which is the convex boundary of some symplectic four-manifold $(W, \omega)$. Then, any Giroux two-handle $W_{0}: Y \longrightarrow Y_{0}$ can be completed to give a compact symplectic manifold $(V, \omega)$ with concave boundary $\partial(V, \omega)=(Y, \xi)$, so that $\omega$ extends smoothly over $X=W \cup_{Y} V$.

Although Eliashberg's is the construction we need, concave fillings have been constructed previously in a number of different contexts, see for example 22], 1], 7], 10, 25]. Indeed, since the first posting of the present article, Etnyre pointed out to us an alternate proof of Eliashberg's theorem [6], see also [25].

In the construction, $V$ is given as the union of the Giroux two-handle with a surface bundle $V_{0}$ over a surface-with-boundary which extends the fiber bundle structure over $Y_{0}$. Moreover, the fibers of $V_{0}$ are symplectic. By forming a symplectic sum if necessary, one can arrange for $b_{2}^{+}(V)$ to be arbitrarily large.

To state the stronger non-vanishing theorem, we use a refinement of the contact element using twisted coefficients. We can repeat the construction of $c(\xi)$ with 
coefficients in any module $M$ over $\mathbb{Z}\left[H^{1}(Y ; \mathbb{Z})\right]$ (compare Remark 4.5 of [30]), to get an element

$$
c(\xi ; M) \in \underline{\widehat{H F}}(Y ; M) / \mathbb{Z}\left[H^{1}(Y ; \mathbb{Z})\right]^{\times} .
$$

As the notation suggests, this is an element $c(\xi ; M) \in \underline{\widehat{H F}}(Y ; M)$, which is welldefined up to overall multiplication by a unit in the group-ring $\mathbb{Z}\left[H^{1}(Y ; \mathbb{Z})\right]$. Let $c^{+}(\xi ; M)$ denote the image of $c(\xi ; M)$ under the natural map $\underline{\widehat{H F}}(-Y ; M) \longrightarrow$ $\underline{H F}^{+}(-Y ; M)$, and let $c_{\text {red }}^{+}(\xi ; M)$ denote its image under the projection $\underline{H F}^{+}(-Y ; M) \longrightarrow \underline{H F^{+}}+(-Y ; M)$.

In our applications, we will typically take the module $M$ to be $\mathbb{Z}[\mathbb{R}]$, with the action specified by some two-form $\omega$ over $Y$, so that we get $c(\xi ;[\omega]) \in$ $\underline{\underline{H F}}(-Y ;[\omega])$. The following theorem should be compared with a theorem of Kronheimer and Mrowka [17, see also Section 6 of [19]:

Theorem 4.2 Let $(W, \omega)$ be a weak filling of a contact structure $(Y, \xi)$. Then, the associated contact invariant $c(\xi ;[\omega])$ is non-trivial. Indeed, it is non-torsion and primitive (as is its image in $\mathrm{HF}^{+}(Y ;[\omega])$. Indeed, if $(W, \omega)$ is a weak-semifilling of $(Y, \xi)$ with disconnected boundary or $(W, \omega)$ is a weak filling of $Y$ with $b_{2}^{+}(W)>0$, then the reduced invariant $c_{\text {red }}^{+}(\xi ;[\omega])$ is non-trivial (and indeed non-torsion and primitive).

Proof Let $(W, \omega)$ be a symplectic filling of $(Y, \xi)$ with convex boundary.

Consider Eliashberg's cobordism bounding $Y, V=W_{0} \cup_{Y_{0}} V_{0}$, where here $W_{0}: Y \longrightarrow Y_{0}$ is the Giroux two-handle and $V_{0}$ is a surface bundle over a surface-with-boundary. Now, the union

$$
X=V_{0} \cup_{-Y_{0}} \cup W_{0} \cup_{-Y} W
$$

is a closed, symplectic four-manifold. (As the notation suggests, we have "turned around" $W_{0}$, to think of it as a cobordism from $-Y_{0}$ to $-Y$; similarly for $V_{0}$.) Arrange for $b_{2}^{+}\left(V_{0}\right)>1$, and decompose $V_{0}$ further by introducing an admissible cut by $N$. Now, $N$ decompose $X$ into two pieces $X=X_{1} \cup_{N} X_{2}$, where $b_{2}^{+}\left(X_{i}\right)>0$, and we can suppose now that $X_{2}$ contains the Giroux cobordism, i.e.

$$
X_{2}=\left(V_{0}-X_{1}\right) \cup_{-Y_{0}} \cup W_{0} \cup_{-Y} W .
$$

Now, by the definition of $\Phi$, for any given $\mathfrak{s} \in \operatorname{Spin}^{c}(X)$, there is an element $\theta \in H F^{+}\left(N,\left.\mathfrak{s}\right|_{N}\right)$ with the property that

$$
\Phi_{X, \mathfrak{s}}=F_{X_{2}-B_{2}}^{+}(\theta) .
$$


(By definition of $\Phi$, the element $\theta$ here is any element of $\mathrm{HF}^{+}\left(N,\left.\mathfrak{s}\right|_{N}\right)$ whose image under the connecting homomorphism in the second exact sequence in Equation (11) coincides with the image of a generator of $H F^{-}\left(S^{3}\right)$ under the map $F_{X_{1}-B_{1}}^{-}: H F^{-}\left(S^{3}\right) \longrightarrow H F^{-}\left(N,\left.\mathfrak{s}\right|_{N}\right)$.) Applying the product formula for the decomposition of Equation (4), we get that

$$
\sum_{\eta \in H^{1}(Y ; \mathbb{Z})} \Phi_{X, \mathfrak{k}(\omega)+\delta \eta}=F_{W-B_{2}}^{+} \circ F_{W_{0}}^{+} \circ F_{V_{0}-X_{1}}^{+}(\theta) .
$$

In terms of $\omega$-twisted coefficients, we have that

$$
\sum_{\eta \in H^{1}\left(Y_{0} ; \mathbb{Z}\right)} \Phi_{X, \mathfrak{k}(\omega)+\delta \eta} \cdot T^{\left\langle\omega \cup c_{1}(\mathfrak{k}(\omega)+\delta \eta),[X]\right\rangle}=\underline{F}_{W-B_{2} ;[\omega]}^{+} \circ \underline{F}_{W_{0} ;[\omega]}^{+} \circ \underline{F}_{V_{0}-X_{1} ;[\omega]}^{+}(\underline{\theta}) \text {. }
$$

(Here, $\underline{\theta} \in \underline{H F^{+}}\left(N,\left.\mathfrak{s}\right|_{N} ;[\omega]\right)$ is the analogue of the class $\theta$ considered earlier.) But $H F^{+}\left(Y_{0}, \mathfrak{t}\right) \cong \mathbb{Z}[\mathbb{R}]$ is generated by $c_{0}^{+}(\pi)$ (where here $\pi: Y_{0} \longrightarrow S^{1}$ is the projection obtained from restricting the bundle structure over $V_{0}$, and $\mathfrak{t}$ is the restriction of $\mathfrak{k}(\omega)$ to $\left.Y_{0}\right)$, so there is some element $p(T) \in \mathbb{Z}[\mathbb{R}]$ with the property that $\underline{F}_{V_{0}-\operatorname{nd}(F)}^{+}(\underline{\theta})=p(T) \cdot c^{+}(\pi)$. Thus,

$$
\sum_{\eta \in H^{1}\left(Y_{0} ; \mathbb{Z}\right)} \Phi_{X, \mathfrak{k}(\omega)+\delta \eta} \cdot T^{\left\langle\omega \cup c_{1}(\mathfrak{k}(\omega)+\delta \eta),[X]\right\rangle}=p(T) \cdot \underline{F}_{W-B_{2}}^{+}\left(c^{+}(\xi ;[\omega])\right) .
$$

The left-hand-side here gives a polynomial in $T$ (well defined up to an overall sign and multiple of $T$ ) whose lowest-order term is one, according to Theorem 1.1 of [34] (recalled in Section 3). It follows at once that $\underline{F}_{W-B_{2}}^{+}\left(c^{+}(\xi ;[\omega])\right)$ is non-trivial. Indeed, it also follows that $\underline{F}_{W-B_{2}}^{+}\left(c^{+}(\xi ;[\omega])\right)$ is a primitive homology class (since the leading coefficient is 1 ), and no multiple of it zero. This implies the same for $c(\xi ;[\omega])$.

Now, when $b_{2}^{+}(W)>0$, we use $Y$ as a cut for $X$ to show that the induced element $c_{\text {red }}^{+}(\xi ;[\omega])$ is non-trivial (primitive and torsion). In the case where $Y$ is semi-fillable with disconnected boundary, we can close off the remaining boundary components as in Theorem 4.1 to construct a new symplectic filling $W^{\prime}$ of $Y$ with one boundary component and $b_{2}^{+}\left(W^{\prime}\right)>0$, reducing to the previous case.

Proof of Theorem 1.4 A three-manifold $Y$ is an $L$-space if it is a rational homology three-sphere and $\widehat{H F}(Y)$ is a free $\mathbb{Z}$-module of rank $\left|H_{1}(Y ; \mathbb{Z})\right|$. Note that for an $L$-space, $H F_{\text {red }}^{+}(Y) \otimes_{\mathbb{Z}} \mathbb{Q}=0$. This is an easy application of the long exact sequence (11), together with the fact that the the intersection of the kernel of $U: H F^{+}(Y) \longrightarrow H F^{+}(Y)$ with the image of $H F^{\infty}(Y)$ inside $H F^{+}(Y)$ has rank $\left|H_{1}(Y ; \mathbb{Z})\right|$, since $H F^{\infty}(Y) \cong \mathbb{Z}\left[U, U^{-1}\right]$ (cf. Theorem 10.1 
of [26]), the map from $H F^{\infty}(Y)$ to $H F^{+}(Y)$ is an isomorphism in all sufficiently large degrees (i.e. $U^{-n}$ for $n$ sufficiently large), and it is trivial in all sufficiently small degrees.

For a three-manifold $Y$ with $b_{1}(Y)=0, \underline{H F^{+}}(Y ;[\omega]) \cong H F^{+}(Y) \otimes_{\mathbb{Z}} \mathbb{Z}[\mathbb{R}]$, since $[\omega] \in H^{2}(Y ; \mathbb{Q})$ is exact. Thus, the reduced group in which $c_{\text {red }}^{+}(\xi ;[\omega])$ lives consists only of torsion classes, and the result now follows from Theorem 4.2

Sometimes, it is easier to use $\mathbb{Z} / p \mathbb{Z}$ coefficients (especially when $p=2$ ). To this end, we say that $Y$ a rational homology three-sphere is a $\mathbb{Z} / p \mathbb{Z}-L$-space for some prime $p$ if $\widehat{H F}\left(Y ; \mathbb{Z} / p \mathbb{Z}\right.$ ) has rank $\left|H_{1}(Y ; \mathbb{Z})\right|$ over $\mathbb{Z} / p \mathbb{Z}$ (of course, an $L$ space is automatically a $\mathbb{Z} / p \mathbb{Z}-L$-space for all $p)$. Since $c^{+}(\xi ;[\omega])$ is primitive, the above argument shows that a $\mathbb{Z} / p \mathbb{Z}-L$-space (for any prime $p$ ) cannot support a taut foliation.

The need to use twisted coefficients in the statement of Theorem 4.2 is illustrated by the three-manifold $Y$ obtained as zero-surgery on the trefoil. The reduced Heegaard Floer homology with untwisted coefficients is trivial (cf. Equation 26 of [32]), but this three-manifold admits a taut foliation. (In particular the reduced Heegaard Floer homology of this manifold with twisted coefficients is non-trivial, cf. Lemma 8.6 of 32 .)

\section{The Thurston norm}

We turn our attention to the proof of Theorem 1.1

Proof of Theorem 1.1] It is shown in Section 1.6 of [26] that if $\underline{\widehat{H F}}(Y, \mathfrak{s}) \neq 0$, then

$$
\left|\left\langle c_{1}(\mathfrak{s}), \xi\right\rangle\right| \leq \Theta(\xi) .
$$

(The result is stated there for $\mathrm{HF}^{+}$with untwisted coefficients, but the argument there applies to the case of $\underline{\widehat{H F}}$.) It remains to prove that if $\Sigma \subset Y$ is an embedded surface which minimizes complexity in its homology class $\xi$, then there is a $\operatorname{Spin}^{c}$ structure $\mathfrak{s}$ with $\underline{\widehat{H F}}(Y, \mathfrak{s}) \neq 0$ and

$$
\left\langle c_{1}(\mathfrak{s}),[\Sigma]\right\rangle=-\chi_{+}(\Sigma) \text {. }
$$

The Künneth principle for connected sums (cf. Theorem 1.5 of [26]) states that

$$
\underline{\widehat{H F}}\left(Y_{1} \# Y_{2}, \mathfrak{s}_{1} \# \mathfrak{s}_{2}\right) \otimes_{\mathbb{Z}} \mathbb{Q} \cong \underline{\widehat{H F}}\left(Y_{1}, \mathfrak{s}_{1}\right) \otimes_{\mathbb{Z}} \underline{\widehat{H F}}\left(Y_{2}, \mathfrak{s}_{2}\right) \otimes_{\mathbb{Z}} \mathbb{Q} .
$$

In particular, if $\underline{\widehat{H F}}\left(Y_{1}, \mathfrak{s}_{1}\right) \otimes_{\mathbb{Z}} \mathbb{Q}$ and $\underline{\widehat{H F}}\left(Y_{2}, \mathfrak{s}_{2}\right) \otimes_{\mathbb{Z}} \mathbb{Q}$ are non-trivial, then so is $\underline{\widehat{H F}}\left(Y_{1} \# Y_{2}, \mathfrak{s}_{1} \# \mathfrak{s}_{2}\right) \otimes_{\mathbb{Z}} \mathbb{Q}$. Since every closed three-manifold admits a 
connected sum decomposition where the summands are all either irreducible or copies of $S^{2} \times S^{1}\left[23\right.$, it suffices to verify that $\underline{\widehat{H F}}(Y, \mathfrak{s}) \otimes_{\mathbb{Z}} \mathbb{Q}$ is nontrivial for the elementary summands of $Y$. (It is straightforward to see that $\Theta_{Y_{1} \# Y_{2}}\left(\xi_{1}+\xi_{2}\right)=\Theta_{Y_{1}}\left(\xi_{1}\right)+\Theta_{Y_{2}}\left(\xi_{2}\right)$ in $Y_{1} \# Y_{2}$, where here $\xi_{i} \in H_{2}\left(Y_{i}\right)$, under the natural identification $H_{2}\left(Y_{1} \# Y_{2}\right) \cong H_{2}\left(Y_{1}\right) \oplus H_{2}\left(Y_{2}\right)$.)

We first observe that if $Y$ has trivial Thurston semi-norm (for example, when $b_{1}(Y)=0$ or $\left.Y=S^{2} \times S^{1}\right)$, then there is an element $\mathfrak{s} \in \operatorname{Spin}^{c}(Y)$ for which $\widehat{H F}(Y, \mathfrak{s}) \neq 0$. Indeed, it is shown in Theorem 10.1 of $\left[26\right.$ ] that $\underline{H F^{\infty}}(Y, \mathfrak{s}) \cong$ $\mathbb{Z}\left[U, U^{-1}\right]$ for any $\mathfrak{s}$ with $c_{1}(\mathfrak{s})=0$. Also, for such Spin ${ }^{c}$ structures, the map from $\underline{H F} \underline{F}^{\infty}(Y, \mathfrak{s})$ to $\underline{H F}{ }^{+}(Y, \mathfrak{s})$ is non-trivial. The non-triviality of $\underline{\hat{H F}}(Y, \mathfrak{s})$ follows at once (using the analogue of Exact Sequence (11) for the case of twisted coefficients).

In the case where $Y$ is an irreducible three-manifold with non-trivial Thurston norm, and $\Sigma$ is a surface which minimizes complexity in its homology class, Gabai [8] constructs a smooth taut foliation $\mathcal{F}$ for which

$$
\left\langle c_{1}(\mathcal{F}),[\Sigma]\right\rangle=-\chi_{+}(\Sigma) .
$$

According to a theorem of Eliashberg and Thurston, then $[-1,1] \times Y$ can be equipped with a convex symplectic form, which extends $\mathcal{F}$, thought of as a foliation over $\{0\} \times Y$. In particular, their result gives a weakly symplectically semi-fillable contact structure $\xi$ with $\left\langle c_{1}(\xi),[\Sigma]\right\rangle=-\chi_{+}(\Sigma)$. It follows now from Theorem 4.2 that $c(\xi,[\omega]) \in \underline{\widehat{H F}}(Y,[\omega], \mathfrak{s}(\xi)) \otimes_{\mathbb{Z}} \mathbb{Q} \neq 0$.

One approach to Theorem 1.2 would directly relate knot Floer homology with the twisted Floer homology of the zero-surgery. We opt, however, to give an alternate proof which uses the relation between the knot Floer homology and the Floer homology of the zero-surgery in the untwisted case, and adapts the proof rather than the statement of Theorem 1.1. The relevant relationship between these groups can be found in Corollary 4.5 of [31, according to which if $d>1$ is the smallest integer for which $\widehat{H F K}(K, d) \neq 0$, then

$$
\widehat{H F K}(K, d) \cong H F^{+}\left(S_{0}^{3}(K), d-1\right),
$$

where here we have identified $\operatorname{Spin}^{c}\left(S_{0}^{3}(K)\right) \cong \mathbb{Z}$ by the map $\mathfrak{s} \mapsto\left\langle c_{1}(\mathfrak{s}),[\Sigma]\right\rangle / 2$, where $[\Sigma] \in H_{2}\left(S_{0}^{3}(K) ; \mathbb{Z}\right) \cong \mathbb{Z}$ is some generator. (Note that the choice of generator is not particularly important, as $H^{+}\left(S_{0}^{3}(K), i\right) \cong H F^{+}\left(S_{0}^{3}(K),-i\right)$, according to the conjugation invariance of Heegaard Floer homology, Theorem 2.4 of $[26]$.)

This result will be used in conjunction with the "adjunction inequality" for knot Floer homology, Theorem 5 of [31], which shows that $\widehat{H F K}(K, i)=0$ for 
all $|i|>g(K)$; and indeed, the proof of that result proceeds by constructing a compatible doubly-pointed Heegaard diagram (from a genus-minimizing Seifert surface for $K$ ) which has no simultaneous trajectories $\mathbf{x}$ with $s(\mathbf{x})>g(K)$.

Proof of Theorem 1.2 Let $K \subset S^{3}$ be a knot with genus $g$. Assume for the moment that $g>1$. Let $Y$ be the three-manifold obtained as zero-framed surgery on $S^{3}$ along $K$, and let $[\Sigma] \in H_{2}(Y ; \mathbb{Z})$ denote a generator. In this case, Gabai [9] constructs a taut foliation $\mathcal{F}$ over $Y$ with $\left\langle c_{1}(\mathcal{F}),[\Sigma]\right\rangle=2-2 g$. Eliashberg's theorem [3] now provides a symplectic four-manifold $X=X_{1} \cup_{Y}$ $X_{2}$, where here $b_{2}^{+}\left(X_{i}\right)>0$. According to the product formula Equation (2), the sum

$$
\sum_{\eta \in H^{1}(Y)} \Phi_{X, \mathfrak{k}(\omega)+\delta \eta}
$$

is calculated by a homomorphism which factors through the Floer homology $H F^{+}\left(Y,\left.\mathfrak{k}(\omega)\right|_{Y}\right)$. On the other hand, $c_{1}(\mathfrak{k}(\omega))$ gives a cohomology class whose evaluation on a generator for $H_{2}(Y ; \mathbb{Z}$ ) is non-trivial when $g>1$ (for a suitable generator, this evaluation is given by $2-2 g$ ). Since the image of a generator of $H^{1}(Y ; \mathbb{Z})$ is represented by a surface in $X$ with square zero and non-zero evaluation of $c_{1}(\mathfrak{s}(\omega))$, it follows that the various terms in the sum are homogeneous of different degrees. But by Theorem 1.1 of [34, it follows that the term corresponding to $\mathfrak{k}(\omega)$ (and hence the sum) is non-trivial. It follows now that $H F^{+}\left(Y,\left.\mathfrak{k}(\omega)\right|_{Y}\right)=H F^{+}\left(S_{0}^{3}(K), g-1\right)$ (for suitably chosen generator) is non-trivial and hence, in view of Equation (7), Theorem 1.2 follows for knots with genus at least two.

Suppose that $g=1$. In this case, we have a Künneth principle for the knot Floer homology (cf. Equation 5 of [31]), according to which (since $\widehat{H F K}(K, s)=0$ for all $s>1$ ),

$$
\widehat{H F K}(K \# K, 2) \otimes_{\mathbb{Z}} \mathbb{Q} \cong \widehat{H F K}(K, 1) \otimes_{\mathbb{Q}} \widehat{H F K}(K, 1) .
$$

But $K \# K$ is a knot with genus 2 , and hence $\widehat{H F K}(K \# K, 2)$ is non-trivial; and hence, so is $\widehat{H F K}(K, 1)$.

Proof of Corollary $\mathbf{1 . 3}$ According to the integral surgeries long exact sequence for Heegaard Floer homology (in its graded form), if $S_{p}^{3}(K) \cong L(p, 1)$, the Alexander polynomial of $K$ is trivial (indeed $H F^{+}\left(S_{0}^{3}(K)\right) \cong H F^{+}\left(S^{2} \times\right.$ $\left.S^{1}\right)$ ), cf. Theorem 1.8 of [32]. In [29], it is shown that if $S_{p}^{3}(K)$ is a lens space for some integer $p$, then the knot Floer homology $\widehat{H F K}_{*}(K, *)$ is determined by the Alexander polynomial $\Delta_{K}(T)$ (cf. Theorem 1.2 of [29]) which in the present case is trivial. Thus, in view of Theorem 1.2, the knot $K$ is trivial. 
Proof of Corollary [1.5 In the proof of Theorem 5 of [31, we demonstrate that if a knot has genus $g$, then there is a compatible Heegaard diagram with no simultaneous trajectories $\mathbf{x}$ for which $s(\mathbf{x})>g$. In the opposite direction, note that $\widehat{H F K}(K, d)$ is generated by simultaneous trajectories with $s(\mathbf{x})=$ $d$. According to Theorem $1.2 \widehat{H F K}(K, g) \neq 0$, and hence any compatible Heegaard diagram must contain some simultaneous trajectories $\mathbf{x}$ with $s(\mathbf{x})=$ $g$.

\section{References}

[1] S Akbulut, B Ozbagci, Lefschetz fibrations on compact Stein surfaces, Geom. Topol. 5 (2001) 319-334

[2] S K Donaldson, Lefschetz pencils on symplectic manifolds, J. Differential Geom. 53 (1999) 205-236

[3] Y M Eliashberg, A few remarks about symplectic filling, Geom. Topol. 8 (2004) 277-293

[4] Y M Eliashberg, M Gromov, Convex symplectic manifolds, from: "Several complex variables and complex geometry, Part 2 (Santa Cruz, CA, 1989)", Proc. Sympos. Pure Math. 52, Amer. Math. Soc. Providence, RI (1991) 135-162

[5] Y M Eliashberg, W P Thurston, Confoliations, University Lecture Series 13, American Mathematical Society (1998)

[6] J B Etnyre, On symplectic fillings, Algebr. Geom. Topol. 4 (2004) 73-80

[7] J B Etnyre, K Honda, On symplectic cobordisms, Math. Ann. 323 (2002) 31-39

[8] D Gabai, Foliations and the topology of 3-manifolds, J. Differential Geom. 18 (1983) 445-503

[9] D Gabai, Foliations and the topology of 3-manifolds III, J. Differential Geom. 26 (1987) 479-536

[10] D T Gay, Explicit concave fillings of contact three-manifolds, Math. Proc. Cambridge Philos. Soc. 133 (2002) 431-441

[11] E Giroux, Géométrie de contact: de la dimension trois vers les dimensions supérieures, from: "Proceedings of the International Congress of Mathematicians, Vol. II (Beijing, 2002)", Higher Ed. Press, Beijing (2002) 405-414

[12] R E Gompf, A I Stipsicz, 4-manifolds and Kirby calculus, volume 20 of Graduate Studies in Mathematics, American Mathematical Society, Providence, RI (1999)

[13] C McA Gordon, Some aspects of classical knot theory, from: "Knot theory (Proc. Sem. Plans-sur-Bex, 1977)", Lecture Notes in Math. 685, Springer-Verlag (1978) 1-60 
[14] C McA Gordon, J Luecke, Knots are determined by their complements, J. Amer. Math. Soc. 2 (1989) 371-415

[15] L H Kauffman, On knots, Annals of Mathematics Studies 115, Princeton University Press (1987)

[16] P B Kronheimer, T S Mrowka, Floer homology for Seiberg-Witten Monopoles, in preparation

[17] P B Kronheimer, T S Mrowka, Monopoles and contact structures, Invent. Math. 130 (1997) 209-255

[18] P B Kronheimer, TS Mrowka, Scalar curvature and the Thurston norm, Math. Res. Lett. 4 (1997) 931-937

[19] P B Kronheimer, T S Mrowka, P S Ozsváth, Z Szabó, Monopoles and lens space surgeries, arXiv:math.GT/0310164

[20] P Lisca, Symplectic fillings and positive scalar curvature, Geom. Topol. 2 (1998) 103-116

[21] P Lisca, A Stipsicz, Seifert fibered contact three-manifolds via surgery, arXiv:math.SG/0307341

[22] P Lisca, G Matić, Tight contact structures and Seiberg-Witten invariants, Invent. Math. 129 (1997) 500-525

[23] J Milnor, A unique decomposition theorem for 3 -manifolds, Amer. J. Math. 84 (1962) 1-7

[24] A Némethi, On the Ozsváth-Szabó invariant of negative definite plumbed 3 manifolds, arXiv:math.GT/0310083

[25] H Ohta, K Ono, Simple singularities and topology of symplectically filling 4-manifold, Commen. Math. Helv. 74 (1999) 575-590

[26] P S Ozsváth, Z Szabó, Holomorphic disks and three-manifold invariants: properties and applications, arXiv:math.SG/0105202, to appear in Annals of Math.

[27] P S Ozsváth, Z Szabó, Holomorphic disks and topological invariants for closed three-manifolds, arXiv:math.SG/0101206, to appear in Annals of Math.

[28] P S Ozsváth, Z Szabó, Holomorphic triangles and invariants for smooth fourmanifolds, arXiv:math.SG/0110169

[29] P S Ozsváth, Z Szabó, On knot Floer homology and lens space surgeries, arXiv:math.GT/0303017

[30] P S Ozsváth, Z Szabó, Heegaard Floer homologies and contact structures, arXiv:math.SG/0210127

[31] PS Ozsváth, Z Szabó, Holomorphic disks and knot invariants, (2002) arXiv:math.GT/0209056

[32] P S Ozsváth, Z Szabó, Absolutely graded Floer homologies and intersection forms for four-manifolds with boundary, Advances in Mathematics 173 (2003) 179-261 
[33] P S Ozsváth, Z Szabó, Heegaard Floer homology and alternating knots, Geom. Topol. 7 (2003) 225-254

[34] P S Ozsváth, Z Szabó, Holomorphic triangle invariants and the topology of symplectic four-manifolds, Duke Math. J. 121 (2004) 1-34

[35] J Rasmussen, Floer homology and knot complements, Ph.D. thesis, Harvard University (2003)

[36] C H Taubes, The Seiberg-Witten invariants and symplectic forms, Math. Res. Lett. 1 (1994) 809-822

[37] C H Taubes, More constraints on symplectic forms from Seiberg-Witten invariants, Math. Res. Lett. 2 (1995) 9-13

[38] C H Taubes, Seiberg Witten and Gromov invariants for symplectic 4-manifolds, volume 2 of First International Press Lecture Series, International Press, Somerville, MA (2000), edited by Richard Wentworth

[39] W P Thurston, A norm for the homology of 3-manifolds, Mem. Amer. Math. Soc. 59 (1986) i-vi and 99-130

[40] V Turaev, Torsion invariants of $\operatorname{Spin}^{c}$-structures on 3 -manifolds, Math. Res. Lett. 4 (1997) 679-695

[41] E Witten, Monopoles and four-manifolds, Math. Res. Lett. 1 (1994) 769-796 